\title{
Physical Entities and Mathematical Representation
}

\author{
Chester H. Page
}

\begin{abstract}
(August 22, 1961)
Certain basic postulates about physical observables yield the structure of their mathematical representation. Measure equations are contrasted with quantity equations, and measurement units with abstract units. The abstract vector spaces in which observables are represented comprise the core of dimensional analysis.

Systems of equations, units, and dimensions are discussed, along with comments on rationalization. The problem of assigning a dimension to angle is discussed, and a new proposal offered.
\end{abstract}

Physical systems and experimental situations are quantitatively described in terms of "observables", entities which are subject to measurement. These entities are of various kinds, e.g., force, mass, charge, etc. Two entities are said to be of the same kind if they can be compared, i.e., if the question, $E_{1} \leq E_{2}$ ?, is meaningful. For example, we can compare one force with another to determine which is larger, but we cannot say whether a given distance is larger or smaller than a particular mass.

The implication of the above remarks is that physical entities can be sorted into ordered chains, or one-dimensional sets, of like entities:

$$
\begin{aligned}
& q_{1} \leq q_{2} \leq q_{3} \ldots . \\
& p_{1} \leq p_{2} \leq p_{3} \ldots .
\end{aligned}
$$

The entities in a chain are said to be of the same "kind"; entities in different chains cannot be compared, and are of different kinds. The common property of entities in the q-chain may conveniently be called "q-ness", for an identifying label.

Observables are not only subject to ordering in sets, but are also subject to measurement. Thus, we can experimentally determine a ratio between two entities in a given chain:

$$
\text { " } q_{2} \text { is } x \text { times as large as } q_{1} " \text {. }
$$

The experimental procedures of physical science are based on the (arbitrary) choice of a particular sample of an entity as a measurement unit for entities of that kind. The general sample of that kind is then described by the statement:

$$
\text { " } q \text { is }\{q\} \text { times as large as } q u "
$$

where $q_{u}$ is a symbol for the particular sample chosen to be the measurement unit, and the number, $\{q\},{ }^{1}$ is called the value, or measure, of the sample, $q$. (In contrast, the Moh hardness scale is a familiar example of chain ordering without metric properties.)

If the concept of an observable, $g$, has any "reality", or is to have any quantitative usefulness, the

1 Several types of brackets are used for special symbols. A list of these notations is appended to the text. entity $q$ must have a significance that is independent of our choice of measurement unit. The observable must be invariant to our mode of description. If we change our measurement unit from $q_{u_{1}}$ to $q_{u_{2}}$, where

$$
q_{u_{2}} \text { is } \alpha \text { times as large as } q_{u_{1}} \text {, }
$$

then the measure of any $q$, relative to $q_{u_{2}}$, must satisfy

$$
\{q\}_{2}=\{q\}_{1} / \alpha
$$

where $\{q\}_{1}$ denotes the measure of the same sample of $q$ relative to $q_{u_{1}}$ as measurement unit.

The experimental laws of physics are expressible as proportions among measures of entities, such as

$$
\{q\}=k\{p\}\{s\} /\{r\} \text {. }
$$

The proportionality constant, $k$, depends upon the choice of measurement units for the various entities involved, and upon the configuration of the experimental setup. It is customary to think of the measures on the right as relating to more "elementary" entities, whose measurement units have already been chosen. The measurement unit of the "new" entity $q$ is then defined by assigning a convenient value to $k$ for a standardized experimental situation.

As we shall see later, a few measurement units can be arbitrarily chosen as a basis for a set of units. These basic units, together with assigned values for the $k$ 's in the fundamental equations, determine the remaining measurement units "germane" to the system.

It should be noted that, during the development of a science, the factor $k$ includes the effects of experimental variables that have either inadvertently or intentionally been held constant. For example, the interaction forces among current-carrying conductors depend upon the fluid medium filling the laboratory. The permeability of air varies so little with normal environmental changes, that the effect of this variable would not appear in experiments leading to an initial formulation of Ampere's force law. 
It should also be noted that there are hidden dependencies among the $k$ 's. For example, the interaction force between two stationary charges, the interaction force between two current loops, and the relation between current and charge implied by the magnetic effect of a moving charge, are not independent. The three $k$ 's involved satisfy a relation involving the speed of light in the medium in which the experiments are performed. The existence of a relation between a set of $k$ 's and the measure of some physical entity implies that at least one $k$ contains a hidden reference to a physical entity not explicitly referred to in the experimental measure equations.

There is a uniqueness postulate in physics, usually not stated, that is relevant to the completeness of a law. "If $\{q\}$ depends upon $\{p\}$, $\{s\}$, etc., then any entity whose measure depends upon the same variables in the same way, and upon no others, is an entity of the same kind as q." Any apparent violation of this postulate is an indication of hidden "variables" in the proportionality constants. These "variables" may be properties of nature which are beyond our power to change, such as the speed of light in vacuo. This constancy prevents the experimenter from including these variables in any particular law, and also allows the theoretician to distribute the effect of hidden variables in any convenient self-consistent manner.

The development of physics as a science, rather than as a taxonomy of empirical relations, was due to the abstract representation of these relations by mathematical relations among mathematical elements, subject to manipulation by algebraic and differential processes. Maxwell wrote: ${ }^{2}$

"There are two methods of interpreting the equations relating to geometry and other concrete sciences.

"We may regard the symbols which occur in the equation as of themselves denoting lines, masses. times, \&c.; or we may consider each symbol as denoting only the numerical value of the corresponding quantity, the concrete unit to which it is referred being tacitly understood.

"If we adopt the first method we shall often have difficulty in interpreting terms which make their appearance during the calculations. We shall therefore consider all the written symbols as mere numerical quantities, and therefore subject to all the operations of arithmetic during the process of calculation. But in the original equations and the final equations, in which every term has to be interpreted in a physical sense, we must convert every numerical expression into a concrete quantity by multiplying it by the unit of that kind of quantity."'

We next examine the basis of the representation of physical entities (concrete quantities) by mathematical elements, and the structure of the associated mathematics. For simplicity, we shall consider all physical entities as scalars; vectors and tensors can be so described in terms of their components.

Since samples of $q$ can be chain-ordered, these samples can be represented by points in a onedimensional topological space. In addition, the

\footnotetext{
2 Encyclopaedia Britannica, Ninth Edition, VII, 241.
}

measurability of observables implies that this is a metric space; and in particular, the measurability as a ratio to a measurement unit implies a onedimensional abstract vector space. We use the same symbol $q$ to denote both a physical entity and its representative point in the vector space. In its latter role as a mathematical element, the symbolic quantity $q$ is often called a "physical quantity." (This is the reason I have been using the term "entity" for the physical case, to distinguish between an "entity" and its representation by a "quantity".) The duality of the symbol $q$ causes little difficulty; the ambiguity is usually resolved by the context. On the other hand, as we shall see later, substitution of a name for $q$ can cause confusion, such as the use of "ampere" for the name of the measurement unit of current as well as for a mathematical element.

The particular entity sample, $q_{u}$, used for a measurement unit, will be represented by a point denoted by $\langle q\rangle$, the base or unit vector of the space in which the $q$ entity is represented. The vector $\langle q\rangle$ is an abstract or symbolic unit, as contrasted with the more concrete measurement unit. Both abstract and measurement units are often referred to as "units", with no modifier. Thus, to an experimenter, the unit of current is the ampere, and is specified by operational procedures, while to many theorists, the unit of current is also the ampere, but is defined to be a mathematical element!

The relation between an entity sample and its measurement unit, " $q$ is $\{q\}$ times as large as $q_{u}$ ", is represented by the equation

$$
q=\{q\}\langle q\rangle \text {. }
$$

This is an equation relating points or vectors in the space of $q$-elements. Let $[q]$ be a symbol for this $q$-space, the representation space for entities of the kind $q$. We can think of $[q]$ as a symbol for the attribute of " $q$-ness" of the entity. (Later, [q] will also be called the "dimension" of $q$.)

\section{Generalization of Measure Equations}

If $k$ contains no hidden "variables", the theoretician generalizes the experimenter's measure equation, $\{q\}=k\{p\}\{s\}$, to the abstract relation, $q=k p s$, where $q, p$, and $s$ are now mathematical elements in the $[q],[p]$, and $[s]$ spaces. The innocent looking "product", $p s$, is not yet defined. However, our postulate on the completeness of a physical law, in combination with the assumed relation between $q, p$, and $s$, implies that the product operation has the following property:

"The product of any point in $[p]$ and any point in $[s]$ is a point in $[q]$, and conversely, any point in $[q]$ can be expressed as the product of some point in $[p]$ and some point in $[s]$." "We can therefore define $[q]$ to be the product of the spaces $[p]$ and $[s]$ :

$$
[q]=[p][s] .
$$

Since the order of changing the $p$ and $s$ conditions in an experiment is immaterial, we can require the 
above multiplication to be commutative:

$$
[q]=[p][s]=[s][p]=[p s]=[s p] .
$$

Note that we are not here defining the result $[q]$ of the product operation, but are defining the product operation by its result.

We can also define the less frequently needed sum of spaces to be a logical sum. Since $[p]$ is the space of $p$-ness, i.e., the set of all possible values of $p$ (collectively), $[p]+[s]$ can be defined to be the set of all elements possessing $p$-ness plus the set of all elements possessing $s$-ness; i.e., the set of all elements possessing either $p$-ness or $s$-ness (or both). It follows that

$$
\begin{aligned}
& {[p]+[p]+\ldots+[p]=[p]} \\
& n[p]=[p] \\
& {[n]=[m]=[1] \text {; the space of numbers, or }} \\
& \text { "numeric space". }
\end{aligned}
$$

Also

[1] $[p]=[p]$, since any element of [1] multiplied by any element of $[p]$ yields an element in $[p]$.

The self-consistency of the experimental proportionalities of physies implies a unique multiplication table for the various $[q]$, and in fact that the $[q]$ form a group, with [1] as its identity element.

In general, $k$ may involve hidden "variables". The mathematical model must have internal consistency, and to be useful, should satisfy a correspondence principle: The measure equation deduced from a symbolic equation is to be identical with the experimental measure equation being modeled. The measure equation, $\{q\}=k\{p\}\{s\}$, is generalized to

$$
q=\langle\Gamma\rangle k p s
$$

where $\langle\Gamma\rangle$ is a unit vector in an arbitrary space. Since $q \equiv\{q\}\langle q\rangle$, etc., we immediately find the unit equation,

$$
\langle q\rangle=\langle\Gamma\rangle\langle p\rangle\langle s\rangle,
$$

and the space relation,

$$
[q]=[\Gamma][p][s]
$$

The logical necessity for introducing various $[\Gamma]$ spaces was not widely recognized until the MKSA system of units became popular. The dimensional analysis procedures commonly used by MKSA advocates brought to light the hidden "variables" in the conventional proportionality constants.

A constraint on $k^{\prime}$ s, such as $k_{1} k_{2}=\{r\}$, is generalized to

so that

$$
k_{1}\left\langle\Gamma_{1}\right\rangle k_{2}\left\langle\Gamma_{2}\right\rangle=r=\{r\}\langle r\rangle,
$$

$$
\left\langle\Gamma_{1}\right\rangle\left\langle\Gamma_{2}\right\rangle=\langle r\rangle
$$

is the corresponding constraint on the assignment of both the spaces and the unit vectors of the $\Gamma$ 's that are introduced for completeness of the theory.

A set of symbolic units which makes the theoretical and experimental measure equations identical is called a "coherent" set. The absence of coefficients in the resulting unit equations shows that the coherent units form a group, with $\langle 1\rangle$ as the identity. $\langle 1\rangle$ is unity, considered as the unit vector of the space of numerics, [1]. This makes the set of symbolic units "coherent" with the set of corresponding spaces. A set of germane measurement units is represented by a set of coherent symbolic units.

When we treat physics from the symbolic, or quantity calculus, point of view, we consider the quantity equations as basic and the measure equations as derived. The quantity equations are therefore invariant to choice of units. Thus

$$
\{F\}_{1}\langle F\rangle_{1}=\{m\}_{2}\langle m\rangle_{2}\{a\}_{3}\langle a\rangle_{3}
$$

even if the systems 1,2, and 3, are different. For example:

(Measure of force in newtons) times (newton) equals (measure of mass in pounds) times (pound) times (measure of acceleration in centimeters per second) times (centimeter) divided by (second squared).

This equation cannot be factored into

$$
\begin{aligned}
& \{F\}_{1}=\{m\}_{2}\{a\}_{3} \\
& \langle F\rangle_{1}=\langle m\rangle_{2}\langle a\rangle_{3}
\end{aligned}
$$

If $\{m\}_{2}=\alpha\{m\}_{1}$ and $\{a\}_{3}=\beta\{a\}_{1}$, we have

$$
\{F\}_{1}\langle F\rangle_{1}=\alpha \beta\{m\}_{1}\{a\}_{1}\langle m\rangle_{2}\langle a\rangle_{3}
$$

and the conventional measure equation, $\{F\}_{1}=$ $\{m\}_{1}\{a\}_{1}$, yields

$$
\langle F\rangle_{1}=\alpha \beta\langle m\rangle_{2}\langle a\rangle_{3}
$$

In fact, the invariance of the symbolic quantities $m$ and $a$ yields directly:

$$
\langle m\rangle_{1}=\alpha\langle m\rangle_{2} \quad \text { and } \quad\langle a\rangle_{1}=\beta\langle a\rangle_{3}
$$

so that $\langle F\rangle_{1}=\langle m\rangle_{1}\langle a\rangle_{1}$ is a coherent unit equation.

The above noncoherent units yield the measure equation

$$
\{F\}_{1}=\{m\}_{2}\{a\}_{3} / \alpha \beta .
$$

In textbooks, the braces and subscripts are omitted and replaced by marginal notes:

$$
\text { " } E=-10^{-8} d \phi / d t ; E \text { in volts, } \phi \text { in maxwells." }
$$

In any coherent set of units, the equation has the simple form

$$
E=-d \phi / d t .
$$

To summarize, the product equations show the following:

(1) $q=k p s$ defines the product of $p$ and $s$

(2) $[q]=[p][s]$ yields the multiplication table of the group of spaces or dimensions

(3) $\langle q\rangle=\langle p\rangle\langle s\rangle$, or its equivalent

$\{q\}=k\{p\}\{s\}$, yields the multiplication table for the group of coherent units. 


\section{Proportionality Constants}

We have seen that the theoretician must use $k\langle\Gamma\rangle$, or an equivalent, where the experimenter uses a numeric $k$. The conventional way of including the hidden "variable" is to make $k$ a nonnumeric. "This is done by "correcting" the experimenter's equation to

$$
\{q\}=\{k\}\{p\}\{s\}
$$

and using the theoretical equations:

$$
\begin{aligned}
q & =k p s \\
\langle q\rangle & =\langle k\rangle\langle p\rangle\langle s\rangle .
\end{aligned}
$$

A given experimental law, such as Coulomb's Law for electric charges,

$$
\{F\} \propto\left\{q_{1}\right\}\left\{q_{2}\right\} /\left\{r^{2}\right\}
$$

is frequently subjected to different choices of proportionality constant by different writers. To correlate all these choices, and make them yield the same meaning, requires interpreting them as the measure equations relevant to a single symbolic equation, with different choices of units. Thus

yields

$$
F=k q_{1} q_{2} / r^{2}
$$

and

$$
\{\boldsymbol{F}\}\left\{\boldsymbol{r}^{2}\right\}=\{k\}_{i}\left\{q_{1}\right\}_{i}\left\{q_{2}\right\}_{i}
$$

$$
\langle F\rangle\langle r\rangle^{2}=\langle k\rangle_{i}\langle q\rangle_{i}^{2}
$$

in the $i$ th system of coherent units. We assume a common choice of units for $F$ and $r$. Since the left hand sides of these equations are independent of $i$, the choice of unit system, the right hand sides must also be invariant with the choice. Therefore,

$$
\frac{\{q\}_{a}}{\{q\}_{b}}=\sqrt{\frac{\{k\}_{b}}{\{k\}_{a}}}
$$

which agrees with the direct interpretation, by an experimenter, of the change of proportionality constant. But we also have $k=\{k\}_{i}\langle k\rangle_{i}$, which is invariant, so that the change of the unit of $q$ implies a change of the (symbolic) unit of $k$. (Since $\langle k\rangle$ was introduced for theoretical convenience, we do not insist on the existence of a measurement unit, $k_{u}$, corresponding to the symbolic unit $\langle k\rangle$.)

We can interpret the above as follows. The germane measurement unit of an entity $(g)$ depends upon the choice of proportionality constant $(\{k\})$ in the defining equation for the germane unit. If the symbolic quantity $q$ is to have the same meaning in all cases, there must be a corresponding change in the unit of $k$, i.e., in the unit of some real or fictitious entity upon which $k$ depends.

It has earlier been mentioned that the experimental proportionality constant, $\{k\}$, depends upon the geometrical configuration of the defining experiment. When $k$ contains no hidden "variables" in the usual physical sense, we can interpret it to contain a geometrical quantity, represented by some space [k]. The change of $\langle k\rangle$ associated with a change of $\{k\}$ now implies a change of some geometric unit. For example, the magnetizing force at the center of a current circle is

$$
H=k I / r
$$

with the rationalized and non ationalized measures

$$
\begin{aligned}
& \{k\}_{r}=1 / 2 \\
& \{k\}_{n}=2 \pi .
\end{aligned}
$$

If $H$ and $k$ are to have fixed meanings,

yielding

$$
\begin{aligned}
\{H\}_{n}\langle H\rangle_{n} & =\{H\}_{r}\langle H\rangle_{r} \\
\{k\}_{n}\langle k\rangle_{n} & =\{k\}_{r}\langle k\rangle_{r}
\end{aligned}
$$

$$
\frac{\langle k\rangle_{r}}{\langle k\rangle_{n}}=\frac{\{k\}_{n}}{\{k\}_{r}}=4 \pi .
$$

Coherence demands

$$
\begin{aligned}
& \langle H\rangle_{n}=\langle k\rangle_{n}\langle I\rangle /\langle r\rangle \\
& \langle H\rangle_{r}=\langle k\rangle_{r}\langle I\rangle /\langle r\rangle
\end{aligned}
$$

so that

$$
\frac{\langle H\rangle_{r}}{\langle H\rangle_{n}}=\frac{\{H\}_{n}}{\{H\}_{r}}=\frac{\langle k\rangle_{r}}{\langle k\rangle_{n}}=4 \pi .
$$

The relation $\langle k\rangle_{r}=4 \pi\langle k\rangle_{n}$ suggests that $k$ is a solid angle, measured in terms of spheres or steradians as units.

Perucca ${ }^{3,4}$ emphasized that the factor $4 \pi$ appearing in nonrationalized equations of electromagnetism is not a numeric, but the quantity $4 \pi$ steradians. Young ${ }^{5}$ introduced a proportionality constant, $S$, representing the measure of a sphere, into various equations.

This interpretation of rationalization is based on a fixed symbolic equation, with the measures expressed relative to rationalized or nonrationalized units. If, on the other hand, we consider rationalization to be a change of the symbolic equation,

we have

$$
\begin{aligned}
H_{n} & =2 \pi I / r \\
H_{r} & =I / 2 r
\end{aligned}
$$

$$
H_{n}=4 \pi H_{r}
$$

so that $H_{n}$ and $H_{r}$ do not represent the same identical entity. If the factor $4 \pi$ is interpreted as a geometrical quantity, $H_{n}$ and $H_{r}$ are not strictly of the same kind (dimension). We can still satisfy the experimenter, and avoid paradoxes, by arbitrarily setting

$$
\left\{H_{n}\right\}=\{H\}_{n},\left\{H_{r}\right\}=\{H\}_{r}
$$

so that the experimenter need not distinguish between his rationalized measure of $H$ and the measure of a new "rationalized $H$ ". The problem will be discussed in detail in a later section.

\footnotetext{
${ }^{3}$ E. Perucca, Fusione dei sistemi MKSA razionalizzato e non razionalizzato,

French version in La Ricerea Scientifica, p. 1931 (1959).
4 E. Perucca, Solid angle in electromagnetism, rationalization, calculus of ${ }^{4}$ E. Perucca, Solid angle in electromagnetism, rationalization,
quantities, La Ricerca Scientifica 30, Suppl. 12, p. 2200/11 (1960)

quantities, La Ricerca Scientifica 30, Suppl. 12, p. 2200/11 (1960).
5 Leo Young, Electrical units and dimensions, Trans. AIEE 75 , p. 767/71 (1957)
} 


\section{Units and Dimensions}

At any given stage in our knowledge of physics, we have $n$ independent proportionalities involving $N$ kinds of entity. The $n$ proportionalities imply constants $k_{1} \ldots k_{n}$.

We can choose $k_{1} \ldots k_{n}$ as arbitrary numbers, and $N-n$ arbitrary measurement units $(N-n$ prototype standards). These $N-n$ units are called "basic", for they serve as a basis of a unit system whose other germane units are derived from these $N-n$. One of the problems of a standards laboratory, top echelon, is to build experimental set-ups for the realization of the derived units.

We are apt to think of the meter, kilogram, second, degree Kelvin, and candela as the only units defined by prototype standards. The measurement unit for angle is, however, determined by a prototype standard: the circle. Surveyors define the circle to be a realization of 360 units of angle; scientists define it to be a realization of $2 \pi$ units. Similarly, solid angle can be measured in spheres, steradians, or solid degrees.

Note that we made $N$ arbitrary choices in all; $n k$ 's and $N-n$ prototypes. These $N$ choices can be split up differently. For example, we could choose prototype standards for the units of current, voltage, and resistance, and determine the proportionality constant in Ohm's Law experimentally.

We not only have $N$ arbitrary choices for our system of measurement units, but also $N$ choices of representation spaces. If the $k_{1} \ldots k_{n}$ are chosen to be numeric, then we have $n$ unit equations, dimensional equations, or product relations, among $N$ spaces. Hence, $N-n$ spaces can be assigned arbitrary names or dimensional symbols, and the dimensions of the remaining $n$ spaces expressed as products (including reciprocals) of the independent $N-n$. The $N-n$ independent dimensions become the generators of the group of dimensions, and are also often called "basic". "'Basic", throughout, refers to being a "base" rather than implying the possession of any "fundamental" nature.)

The quantity equation appropriate to a given problem is deduced by application of the $n$ basic laws, hence is a combination of the $n$ basic equations. The answer space can readily be computed as an appropriate product of generators. This is the common algebra-checking application of dimensional analysis.

Choosing all $k$ 's to be numeric often discards information. The $q$-ness, or kind, of a quantity uniquely determines its representation space, but if too many spaces are all called numeric, the converse is no longer true, and a dimensional label does not uniquely identify the kind of quantity. For example, torque and energy are usually given a common dimension label, with a trivial attempt at distinction by discriminating between "foot pounds" and "pound feet". This is esthetically distasteful, since torque is work per unit angle. The confusion arises from assigning $[\theta]=[1]$, either for "convenience" or because Maxwell (loc. cit.) referred to "all quantities essentially numerical, such as exponents and exponentials, logarithms, angles, and circular and elliptic functions .. . ."; Maxwell, however, also said (loc. cit.) "By knowing the dimensions of any quantity we are able at once to deduce its numerical value as expressed in terms of one system of units from its numerical value as given in terms of another system."

Langhaar ${ }^{6}$ has paraphrased this: "They are a code for telling us how the numerical value of a quantity changes when the basic units of measurement are subjected to prescribed changes." And later (p. 6)

"The unit of temperature may be assigned independently of the units of the entities of mechanics. Consequently, the symbol $[T],{ }^{7}$ denoting temperature, is regarded as one of the fundamental dimensions. The dimension of an arbitrary variable $z$ is denoted by $[z] . . . . "$

Since the measure of an angle depends upon the choice of unit (e.g., degree or radian), and the choice of unit is independent of the choices made for the other basic units, we conclude that "angle" is not "essentially numerical", but that the dimensions $[\theta]$ and $[\Omega]$ of plane angle and solid angle are ordinarily suppressed or ignored.

In appendix 2 , it is shown that $[\theta]^{2}=[1],[\sin \theta]=[\theta]$, $[\cos \theta]=[1]$, so that $[\theta]=[1]$ is an allowable assignment, but one which is unduly restrictive. More generally, $[\theta]$ is a square root of [1], not further describable. This is analogous to $j^{2}=-1$ in complex numbers, and $i^{2}=j^{2}=k^{2}=-1$ in quaternions. The Gibbs' scalar and vector products carry dimensional implications

so that

$$
\begin{aligned}
{[\cdot] } & =[\cos \theta]=[1] \\
{[X] } & =[\sin \theta]=[\theta]
\end{aligned}
$$

while

so that

$$
W=\mathbf{F} \cdot \mathbf{d} \text { yields }[W]=[F][d]
$$

$$
\mathbf{T}=\mathbf{d} \times \mathbf{F} \text { yields }[T]=[d][\theta][F]
$$

$$
[T]=[W][\theta]
$$

and since

$$
[\theta]^{2}=[1], \quad[T]=[W] /[\theta]
$$

which agrees with our verbal description of torque as work per unit angle.

After we assign $\left\{k_{1}\right\} \ldots\left\{k_{n}\right\}$, determining the germane measurement units, we still have arbitrary choices for $\left[k_{1}\right] \ldots\left[k_{n}\right]$. We can make these explicitly, or implicitly by an arbitrary choice of $[q]$ for the "new" quantity in an equation.

For example, the force between two current elements in vacuo is conventionally considered to be ${ }^{8,9}$

$$
d \mathbf{F}=\frac{\Gamma_{m}}{4 \pi} I_{1} I_{2} \frac{d \mathbf{s}_{2} \times\left(d \mathbf{s}_{1} \times \mathbf{r}\right)}{r^{3}}
$$

The (arbitrary) choice of $\left\{\Gamma_{m}\right\}=4 \pi 10^{-7}$ defines the measurement unit of current in terms of those of force and distance. Classically, $\left[\Gamma_{m}\right]$ is assigned to be [1] in the electromagnetic system of units and

${ }^{6}$ Henry L. Langhaar, Dimensional Analysis and Theory of Models, p. 5 (John Wiley \& Sons, 1959).

${ }^{7}$ The reference uses $\theta$ for temperature; I have changed this to avoid confusion with angle in this discussion.

8 "Conventionally" because it violates Newton's Third Law when applied to incomplete circuits, but not when applied to complete circuits.

9 The symbol, $\Gamma_{m}$, is the "magnetic constant", often denoted by $\mu_{0}$. 
dimensions, yielding $[I]=[m]^{1 / 2}[l]^{1 / 2}[t]^{-1}$. But if we do not assign a space to $\Gamma_{m}$, we can assign an arbitrary space to $I$, yielding: ${ }^{10}$

$$
\left[\Gamma_{m}\right]=\frac{[F]}{[I]^{2}}=\frac{[m][l]}{[I]^{2}[t]^{2}}
$$

This is the customary procedure associated with the MKSA system of units. There are four basic symbolic units (meter, kilogram, second, ampere) associated with four basic dimensions, or generator spaces, but there are still only three basic measurement units. The measurement unit of current (ampere) is defined as a derived unit by assigning a measure to $\Gamma_{m}$. This illustrates the confusion arising from the use of the word "unit" without a modifier; the (measurement) unit of current is a derived unit, but the (symbolic) unit of current is a basic unit. Even worse is the substitution in the above of the name of a unit. The ampere (measurement unit) is derived; the ampere (symbolic unit) is basic!

Any proportionality constant appropriate to an experiment in vacuo can be thought of as a property of space. This interpretation is frequently employed when the phenomenon involves "action at a distance" and the proportionality constant depends upon the intervening medium. 'Thus, in Ampere's force law, the proportionality constant is customarily factored into two terms, $\mu \Gamma_{m}$, where $\mu$ is a numeric (unity for vacuum) representing the differences among media. By virtue of a field interpretation of action at a distance, $\mu$ is thought of as a measure of the permeability of the medium to the field. Hence, we find the usage:

$\mu$ is relative permeability

$$
\begin{gathered}
\mu \Gamma_{m} \text { is "absolute" permeability } \\
\Gamma_{m} \text { is the permeability of space. }
\end{gathered}
$$

The term "permeability of space" implies a property of nature, experimentally determinable, but $\Gamma_{m}$ is arbitrarily assignable! The measure, $\left\{\Gamma_{m}\right\}$, defines the measurement unit of current. The only property of space involved is represented by the "hidden variable", speed of light, which occurs in the constraining relation between two otherwise arbitrary proportionality factors: ${ }^{11} c^{2} \Gamma_{e} \Gamma_{m}=1$.

It is just as logical to interpret the constant, $R$, in the ideal gas law,

$$
p V=R T
$$

as a property of space. Elementary thermodynamics shows us that for any ideal gas, $R=C_{p}-\dot{C}_{v}$, the difference between the specific heat at constant pressure and that at constant volume. Experimentally, $R$ is determined by a sequence of measurements of $p V / T$ at lower and lower gas densities, finally extrapolated to zero density! Does this not yield $\left(C_{p}-C_{v}\right)$ of a vacuum?

\footnotetext{
${ }^{10}$ If we consider the $4 \pi$ in the denominator to be a solid angle,

$$
\left[\Gamma_{m}\right]=\frac{[F][\Omega]}{[I]^{2}} .
$$

The electric constant, $\Gamma_{\bullet}$, is often denoted by $\epsilon_{0}$.
}

The constant $\{R\}$ could have been arbitrarily assigned, thereby defining the measurement unit of 1 temperature. For reasons of convenience, however, the unit of temperature was chosen as basic (determined by a prototype standard), leaving $\{R\}$ as a constant to be measured.

\section{Rationalization}

Coulomb's Law is not a good starting point for a discussion of rationalization, despite the intriguing occurrence of $4 \pi r^{2}$. The introduction of $4 \pi$ into the denominator is compensated by changes of $\Gamma_{e}$ and $\Gamma_{m}$ to keep force unaffected.

We start instead with the electromagnetic equations relevant to the proportionalities:

$$
\begin{aligned}
& \oint \mathbf{H} \cdot d \boldsymbol{\lambda} \propto I \\
& \oint \mathbf{D} \cdot d \boldsymbol{\sigma} \propto q .
\end{aligned}
$$

In particular, the magnetostatic field of a current loop can be expressed in terms of a scalar potential:

$$
\phi \propto I \Omega
$$

where $\Omega$ is the solid angle subtended; at the point of interest, by the current loop. It is convenient to write

and

$$
\phi=-k I \frac{\Omega}{S}
$$

$$
\mathbf{H}=k I \frac{\nabla \Omega}{S}
$$

where $S$ represents the complete solid angle surrounding a point, i.e., the solid angle subtended by any closed surface at any interior point.

The multivalued nature of the solid angle $\Omega$, along any contour linking the loop, yields

$$
\oint \nabla \Omega \cdot d \boldsymbol{\lambda}=S
$$

and therefore

$$
\oint \mathbf{H} \cdot d \boldsymbol{\lambda}=k I .
$$

We also have the geometric relation (see appen$\operatorname{dix} 2$ ),

$$
\{d \Omega\} \propto\{d A\} /\left\{r^{2}\right\}
$$

which implies the quantity equation

$$
d \Omega=\Gamma_{\Omega} d A / r^{2}
$$

without prejudice as to the unit or dimension of $\Omega$. Hence, the angle subtended by the loop is

$$
\Omega=\Gamma_{\Omega} \int \frac{\mathbf{r} \cdot \mathbf{n} d a}{r^{3}}
$$


leading to

$$
\nabla \Omega=\Gamma_{\Omega} \oint \frac{\mathbf{r} \times d \mathbf{l}}{r^{3}}
$$

and

$$
\mathbf{H}=\frac{k I \Gamma_{\Omega}}{S} \oint \frac{\mathbf{r} \times d \mathbf{l}}{r^{3}}
$$

The customary rationalized equations of the form (1), (2), and (3) require, respectively ·

$$
\begin{gathered}
\left\{\frac{k}{S}\right\}=1 / 4 \pi \\
\{k\}=1 \\
\left\{\frac{k \Gamma_{\Omega}}{S}\right\}=1 / 4 \pi
\end{gathered}
$$

so that

$$
\begin{gathered}
\left\{k_{r}\right\}=1 \\
\left\{S_{r}\right\}=4 \pi \\
\left\{\Gamma_{\Omega}\right\}_{r}=1 .
\end{gathered}
$$

The customary nonrationalized equations require

so that

$$
\begin{gathered}
\left\{\frac{k}{S}\right\}=1 \\
\{k\}=4 \pi \\
\left\{\frac{k \Gamma_{\Omega}}{S}\right\}=1
\end{gathered}
$$

$$
\begin{aligned}
\left\{k_{n}\right\} & =4 \pi \\
\left\{S_{n}\right\} & =4 \pi \\
\left\{\Gamma_{\Omega}\right\}_{n} & =1 .
\end{aligned}
$$

Thus in both cases, solid angle is measured in steradians: $\{S\}=4 \pi$, implies $S=4 \pi(\operatorname{str})$, and $\Gamma_{\Omega}$ is a unit vector for accommodating dimensions.

As pointed out by Perucca ${ }^{34}$, the most suitable interpretation of $\left\{k_{n}\right\}=4 \pi$ is $k=4 \pi(\mathrm{str})=S$, or (by eq (2)),

$$
\oint \mathbf{H}_{n} \cdot d \boldsymbol{\lambda}=S I
$$

If we could interpret $\left\{k_{r}\right\}=1$ as $\{S\}_{\text {spheres }}$, then we could identify $H_{r}$ with $H_{n}$, using a change of solid angle unit to explain the relation $\left\{H_{n}\right\}=4 \pi$ $\left\{\boldsymbol{H}_{r}\right\}$. 'This is Young's interpretation ${ }^{5}$ of the effect of rationalization on the coherent unit of solid angle, and would resolve the argument as to whether quantities or units are changed by rationalization, ${ }^{12}$ in favor of the "practical" viewpoint. Unfortunately for this viewpoint, we have already found that $\left\{S_{r}\right\}=4 \pi$ for consistency. If the rationalized form of (2), $\oint \mathbf{H}_{r} \cdot d \boldsymbol{\lambda}=I$, is to be taken as a quantity equation, $[k]=[1]$, and (1) becomes

$$
\mathbf{H}_{r}=\frac{I \nabla \Omega}{S}=\frac{I \nabla \Omega}{4 \pi(\mathrm{str})}
$$

345 See footnotes on p. 230.

${ }_{12}$ F. B. Silsbee, Does rationalization change units?, Elec. Eng. 76, 296/9 (1957). again yielding the Perucca interpretation of the $4 \pi$ factor.

Comparing the rationalized and nonrationalized quantity equations, we see that

$$
H_{n}=S H_{r}=4 \pi(\mathrm{str}) H_{r}
$$

is the quantity change interpretation of rationalization. Since $\langle\Omega\rangle=(\mathrm{str})$ in both systems, there is no ambiguity in taking measures of this relation, finding

$$
\left\{H_{n}\right\}=4 \pi\left\{H_{r}\right\} .
$$

In both systems, eq (3) requires insertion of the unit vector $\Gamma_{\Omega}$ as a factor, if this equation is to be considered a quantity equation. There are three choices:

(a) Consider both forms of eq (3) as measure equations only,

(b) Insert $\Gamma_{\Omega}$ explicitly in writing these equations, or

(c) Require $\left[\Gamma_{\Omega}\right]=[1]$.

Choice (c) makes $[\Omega]=[A] /[l]^{2}=[\theta]$, so that solid angle and plane angle have the same dimension and unit, i.e., solid angle is measured in ordinary radians, or else solid radians and plane radians are both numeric.

The parallel analysis of

$$
\begin{aligned}
\nabla \cdot \mathbf{D} & =k \rho \\
\int \nabla \cdot \mathbf{D} d \tau & =k q=\oint \mathbf{D} \cdot d \boldsymbol{\sigma} \\
\mathbf{D} & =\frac{k \Gamma_{\Omega}}{S} \int \rho \nabla\left(\frac{1}{r}\right) d \tau
\end{aligned}
$$

yields the same conclusions throughout, and the results are compatible with the Maxwell equation for $\nabla \times \mathbf{H}$.

The dimensional conclusions are:

$$
\begin{aligned}
{\left[H_{r}\right] } & =[I] /[l] \\
{\left[H_{n}\right] } & =[\Omega][I] /[l] \\
{\left[D_{r}\right] } & =[q] /[A]=[q] /[\theta][l]^{2} \\
{\left[D_{n}\right] } & =[\Omega][q] /[\theta][l]^{2}=\left[\Gamma_{\Omega}\right][q] /[l]^{2}
\end{aligned}
$$

I wish to thank F. B. Silsbee for many stimulating and valuable discussions.

\section{Notation}

$q$

$\{q\}$

$[q]$

$\langle q\rangle$

(name of unit)
A mathematico-physical quantity, or the corresponding physical entity.

The numerical measure of $q$. If a particular unit system is involved, it is indicated by a subscript outside the bracket.

The abstract vector space of the quantity $q$; also, the "dimension" of $q$.

The element of $[q]$ chosen as a unit vector.

$q_{u} \quad$ The measurement unit of the entity $q$. 


\section{Appendix 1}

The meaning of $\langle p\rangle\langle s\rangle$ cannot be directly interpreted in terms of experimental conditions.

Consider

$$
\begin{aligned}
E & =(1 / 2) m v^{2} \\
\langle E\rangle & =\langle m\rangle\langle v\rangle^{2}
\end{aligned}
$$

The unit equation does not imply that a unit mass moving at unit velocity possesses unit energy. Realizations of measurement units are inferred from measure equations. A system possesses or exhibits the measurement unit of energy, $E_{u}$, when $\{E\}=1$. Now

$$
\{E\}=\{m\}\{v\}^{2} / 2
$$

so that $E_{u}$ is realized by a unit mass moving at a speed which is $\sqrt{2}$ times as big as $v_{u}$, or by a mass twice as big as $m_{u}$, moving at $v_{u}$.

\section{Appendix 2}

Let us choose the radian as the coherent unit of angle, so that the familiar measure equations

$$
\begin{aligned}
& \cos \theta=1-\frac{\theta^{2}}{2 !}+\frac{\theta^{4}}{4 !}-\ldots \\
& \sin \theta=\theta-\frac{\theta^{3}}{3 !}+\frac{\theta^{5}}{5 !}-\ldots
\end{aligned}
$$

can be adopted, without change, as quantity equations. Then

$$
\begin{aligned}
& \cos \theta=1-\frac{\{\theta\}^{2}\langle\theta\rangle^{2}}{2 !}+\ldots \\
& \begin{aligned}
\sin \theta=\{\theta\}\langle\theta\rangle-\frac{\{\theta\}^{3}\langle\theta\rangle^{3}}{3 !}+ & \ldots \\
& =\{\theta\}\langle\theta\rangle\left(1-\frac{\{\theta\}^{2}\langle\theta\rangle^{2}}{3 !}+\ldots\right)
\end{aligned}
\end{aligned}
$$

For each right hand side to represent an element in a single space, rather than in a mixture of spaces, we must have

$$
[\theta]^{2}=[1] .
$$

This implies that any variable $x$ such that its space $[x]$ satisfies $[x]^{2}=[1]$ is an allowable argument for the trigonometric functions. Thus, $\sin \{\theta\}$ is also defined by the infinite series.

Since $\langle\theta\rangle_{\text {conerent }}=($ radian $),(\text { radian })^{2}=\langle 1\rangle$. If we choose a noncoherent unit, say,

then

$$
\langle\theta\rangle=\alpha(\operatorname{radian})^{13}
$$

and

$$
\langle\theta\rangle^{2}=\alpha^{2}
$$

$$
\alpha\{\theta\}=\{\theta\}_{\mathrm{rad}}
$$

13 "(radian)" is not a parenthetical reference to units, but the mathematical element "radian".
This yields

$$
\begin{aligned}
\sin \theta & =\frac{\langle\theta\rangle}{\alpha}\left(\alpha\{\theta\}-\frac{\alpha^{3}\{\theta\}^{3}}{3 !}+\ldots\right) \\
& \equiv \frac{\langle\theta\rangle}{\alpha} \sin \alpha\{\theta\}=\frac{\langle\theta\rangle}{\alpha} \sin \{\theta\}_{\mathrm{rad}}
\end{aligned}
$$

so that

$$
\{\sin \theta\}\langle\sin \rangle=\langle\theta\rangle \sin \{\theta\}_{\mathrm{rad}} / \alpha=(\text { radian }) \sin \{\theta\}_{\mathrm{rad}}
$$

where $\langle\sin \rangle$ is the unit vector in the space where sines are represented.

It follows that $[\sin ]=[\theta]$, and if a given space is to have a unique unit vector, $\langle\sin \rangle=\langle\theta\rangle$. For coherence, the symbolic unit of sine is the radian.

The consequences of $[\theta] \neq[1]$ are interesting. The vector area of a parallelogram is $\mathbf{r}_{1} \times \mathbf{r}_{2}$, and the volume of a parallelopiped is $\mathbf{r}_{1} \times \mathbf{r}_{2} \cdot \mathbf{r}_{3}$. These yield

$$
\begin{aligned}
{[\text { Area }] } & =[l]^{2}[\theta] \\
{[\text { Volume }] } & =[l]^{3}[\theta]
\end{aligned}
$$

which appear odd, but are compatible with the Gauss Divergence Theorem:

$$
\int \nabla \cdot \mathbf{V} d \tau=\oint \mathbf{V} \cdot d \boldsymbol{\sigma}
$$

and Stokes' Theorem:

$$
\int \nabla \times \mathbf{V} \cdot d \boldsymbol{\sigma}=\oint \mathbf{V} \cdot d \boldsymbol{\lambda}
$$

Solid angle is often defined in terms of spherical area by

or its equivalent

$$
d \Omega=d A / r^{2}
$$

$$
d \Omega=\sin \theta d \theta d \phi
$$

With $[$ Area $]=[l]^{2}[\theta] \cdot$ and $[\sin \theta]=[\theta],[\theta]^{2}=[1]$, these formulations agree dimensionally and yield $\langle\Omega\rangle$ $=\langle\theta\rangle=($ radian $)$. The lack of distinction between "solid angle" and "plane angle" is analogous to the lack of distinction between "plane angle" and "numeric" when $d \theta=d l / r$ is used as a quantity equation.

We can avoid all reference to area by considering. a finite acute solid angle bounded by planes (fig. 1). At each edge, the dihedral angle can be expressed in terms of its supplement, $\tau_{i}$, the "turning angle" of the normal to the edge. It is readily shown (in terms of the excess of a spherical triangle) that the measure of the solid angle is proportional to the amount by which $\Sigma_{i} \tau_{i}$ fails to be a complete turn:

$$
\{\Omega\}_{\text {hemlspheres }}=\{\text { U.P.T. }\}_{\text {elre les }}
$$

where U.P.T. is the "uncompleted part of one turn".

The general convention for relating solid angle units to the corresponding plane angle units is that the measure of a hemisphere in solid units is the same 


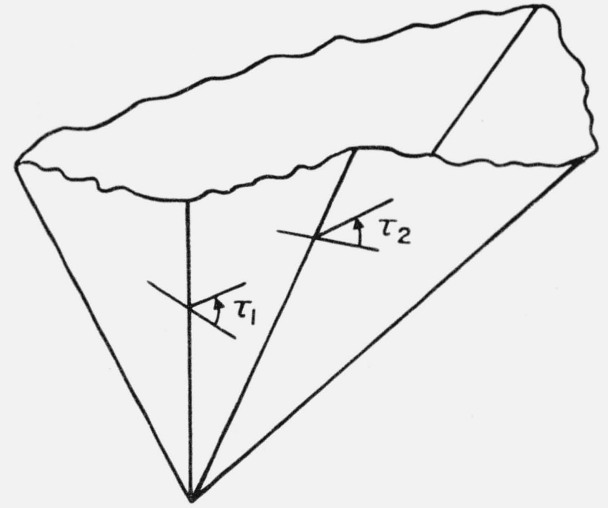

Figure 1

as the measure of a circle in plane units, e.g., a hemisphere is $2 \pi$ steradians or 360 solid degrees. Thus for any unit of plane angle, we define the germane unit of solid angle through

$$
\{\Omega\}_{\text {sold } \theta}=\{\text { U.P.T. }\}_{\text {plane }} \theta
$$

This implies

And therefore

$$
\frac{\Omega}{\langle\Omega\rangle_{i}}=\frac{\text { U.P.T. }}{\langle\theta\rangle_{i}}
$$

$$
\frac{\langle\Omega\rangle_{i}}{\langle\theta\rangle_{i}}=\frac{\Omega}{\text { U.P.T. }}
$$

But the right hand side is invariant to unit choice, therefore $\langle\Omega\rangle_{i}=\Gamma_{\Omega}\langle\theta\rangle_{i}$ with $\Gamma_{\Omega}$ invariant to unit choice. For coherence, $\left\{\Gamma_{\Omega}\right\}=1$, and $\Gamma_{\Omega}$ becomes a unit vector whose only role is to distinguish between solid angle and plane angle.

As shown before, the usual rationalized electromagnetic equations are conveniently interpreted by letting $\Gamma_{\Omega} \equiv 1$, with no dimensional distinction between solid angle and plane angle. This feature is built into the mathematics by using $d \Omega=d A / r^{2}$.

The geometrical definition of sine and cosine as length ratios associated with a right triangle offers a dimensional paradox. It is, however, easily resolved by realizing that we are dealing with a special case of the law of sines:

$$
\frac{l_{1}}{\sin \theta_{1}}=\frac{l_{2}}{\sin \theta_{2}}=\frac{l_{3}}{\sin \theta_{3}}
$$

This equation places no restriction on [sin]. The paradox comes from setting $\sin \pi / 2=\langle 1\rangle$, rather than $\sin \pi / 2=\langle\sin \rangle=\langle\theta\rangle$.

It is also interesting to note that $e^{j \theta}$ is not only two-dimensional in the sense of having both real and imaginary parts, but is also two-dimensional as having components in two spaces:

$$
\begin{aligned}
e^{j \theta} & =\cos \theta+j \sin \theta \\
{[\cos \theta] } & =[1] \\
{[\sin \theta] } & =[\theta] .
\end{aligned}
$$

This is an illustration of the fact that equations used in physics need not be dimensionally homogeneous, when terms arise from the use of an artifice. The form $e^{j \theta}$ does not actually occur in physics; it is introduced with the convention that its real part alone (or its imaginary part alone) represents a variable of interest. Nonartificial exponentials, such as $e^{-w / k T}$, have numeric exponents.

The turning-angle approach to solid angle leads to a formula for the solid angle subtended at a point by an arbitrary closed space curve:

$$
\Omega=2 \pi-\oint r \frac{\mathbf{r} \times \mathbf{r}^{\prime} \cdot \mathbf{r}^{\prime \prime}}{\left(\mathbf{r} \times \mathbf{r}^{\prime}\right) \cdot\left(\mathbf{r} \times \mathbf{r}^{\prime}\right)} d s
$$

where $r^{\prime}=d \mathbf{r} / d s$, and $d s$ is any parameter of progression around the curve.

\section{Appendix 3}

It is interesting to note the relation between "turns" and "radians". Although one complete turn is equivalent to $2 \pi$ radians, i.e., any closed plane curve subtends $2 \pi$ radians at any internal point, the concept of "turn" is topological and not metric. The number of complete passes around any closed path is independent of our concept of angle. But as soon as we try to measure fractional turns, we must define a metric concept for the interpolation and this involves defining a unit for measuring the angle of a partial turn. We must also specify the location of the point around which we are considering the partial turn.

Consider a circular path around a long straight line, as in the circuital law derivation of the magnetic field near a long straight current-carrying conductor. This turn "obviously" involves $2 \pi$ radians. If, however, we consider a nonplanar closed curve, we realize that there are points on the wire for which $\oint d \theta>2 \pi$; the plane angle concept is not really applicable.

In electromagnetic theory, complete encirclements (linkage) and enclosures are involved in the basic concepts and equations. Resolution of total effects into partial effects associated with plane angles and solid angles leads to paradoxes, as in the conventional force law for current elements.

If "turn" is topological and not metric, it should not appear as a dimension or unit. Thus "ampereturn per meter" is a hybrid term; "ampere per meter" should suffice. The inclusion of "turn" in this unit for $H$ is also redundant, since linkage relations and current paths cannot have fractional turns; any linkage of a path with $\chi$ ampere-turns is simply a linkage with $\chi$ amperes. 